\title{
The Challenge of Early Diagnosis of Bacterial Infection in Neonates
}

\section{Bernhard Resch*}

Research Unit for Neonatal Infectious Diseases and Epidemiology, Medical University of Graz, Austria

Neonatal bacterial infection and sepsis are clinical syndromes characterized by systemic signs of infection associated with bacteraemia within the first month of life. There are two patterns of disease with different spectrums of causative agents, early- and late-onset sepsis. Neonatal sepsis still remains a significant cause of morbidity and mortality in the newborn, particularly in preterm, low birth weight infants [1]. Despite advances in neonatal care, overall case-fatality rates from sepsis range from $3 \%$ to as high as $50 \%$ [2]. Clinical signs of bacterial infection are vague and non-specific, and up to now there exists no easily available, reliable marker of infection despite a large bulk of studies focussing on inflammatory indices in neonatology.

In 2002 the International Pediatric Sepsis Consensus Conference was held in San Antonio, Texas with the aim to create clear definitions for the systemic inflammatory response syndrome (SIRS) and different stages of sepsis for children. The definitions should help researchers by creating uniform and standardized entry criteria for clinical trials and, thus, making studies comparable [3]. The panel therefore modified the existing definitions from the American College of Chest Physicians/Society of Critical Care Medicine, published in 1992 [4]. They were designed for the use in adults only and, when revised in 2001 extended with some complements in order to use them also for children [5] The panel' s goal was to create clear definitions of the paediatric sepsis continuum, including SIRS, sepsis, severe sepsis, septic shock and multiple organ dysfunction syndrome, with different criteria for different age groups, ranging from term neonates to adolescents. Despite general acceptance for research purposes and use in various clinical trials, the definitions were said to be sensitive, but not enough specific as screening tools for adaption in the clinical setting [6]. Own data using this definition of SIRS found two-thirds of term newborns and one-third of preterm newborns having been missed in the diagnosis of culture-proven early-onset bacterial infection [7]. Searching for an unambiguous standardized case definition, Modi et al. [8] reported on $\geq 3$ clinical signs demonstrating the best accuracy for a positive blood culture.

The gold standard for the diagnosis of neonatal sepsis is a positive blood culture that obtains results as early as 72 hours. Because there appears to be a sizable subset of neonates who are at risk of sepsis with a colony count less than $4 \mathrm{CFU} / \mathrm{ml}$, then $0.5 \mathrm{ml}$ inoculums of blood into the culture media would be inadequate for sensitive and timely detection of bacteraemia, and one to two milliliters of blood should increase microorganism recovery in the face of low-colonycount sepsis [9]. Recovery of bacteria from a blood culture is often considered to be a "conditio sine qua non" for the diagnosis of neonatal sepsis. Whereas such a strict criterion is certainly appropriate for clinical research, a positive blood culture should not be required for diagnosis of sepsis in everyday practise [10]. Neonates with sepsis may not have any positive cultures, or cultures may be positive only from sites other than blood. Hence it seems to be adequate to divide earlyonset sepsis in culture-proven and clinical sepsis. The latter combines positive clinical signs including two to three different signs of organ involvement (cardiocirculatory, respiratory and gastrointestinal system, neurologic symptoms, fever or hypothermia).with positive maternal factors including maternal fever, prolonged or preterm premature rupture of the membranes or chorioamnionitis and/or positive sepsis markers (inflammatory indices). A recent review of criteria used for the classification of neonatal sepsis found a high disparity in the criteria used with exact specification in only a quarter of studies [11]. Data suggest a false-negative rate of blood cultures of approximately $20 \%$ [10], and therefore, in case of a sick neonate, additional clinical and laboratory findings should be considered before empiric antibiotic therapy is discontinued.

What about haematological tests? Due to the imperfect sensitivity of any single measurement, including the total white cell count, total neutrophil count, and immature-to-total (I:T) neutrophil ratio, combinations led to the development of different scoring systems with divergent results regarding sensitivity, specificity, positive and negative predictive values [10]. Screening blood counts in asymptomatic neonates with maternal risk factors for sepsis are insensitive and show high false-positive rates, and monitoring for development of clinical signs of infection should replace reliance on blood counts [10].

Since its discovery C-reactive-protein (CRP) is one of the standard parameters in the work-up of neonatal sepsis and has been described by Da Silva [12] as probably the best available single test for the diagnosis of neonatal sepsis. Serial determinations of CRP have become "state of the art" for confirmation or exclusion of bacterial infection in neonates. We recently found that CRP was less reliable for early onset of infection in preterm compared to term newborns, with a lower area under the receiver operating characteristics curve and lower median CRP values. Additionally, CRP was associated with non-infectious diagnoses, i.e., meconium aspiration syndrome and application of surfactant, and higher birth weight [13], and preterm infants had lower CRP values compared to term infants, both without symptoms or signs of bacterial infection, suggesting lower cut-off values for preterm infants [14].

An important limitation of CRP is the low sensitivity during the early phases of sepsis. Earlier in the inflammatory cascade activated macrophages release pro-inflammatory cytokines and growth factors. Their increase therefore precedes the changes of CRP. Of the many mediators studied, much attention has been focused on interleukins (IL6, IL8) and cytokines (e.g. tumor-necrosis-factor- $\alpha$ ). Procalcitonin (PCT) is an acute-phase reactant which has the advantage of increasing rapidly after contact to bacterial endotoxin with levels rising after four hours and peaking at six to eight hours. In a recent meta-analysis

*Corresponding author: Dr. Bernhard Resch, Research Unit for Neonatal Infectious Diseases and Epidemiology, Division of Neonatology, Department of Paediatrics, Medical University of Graz, Austria, Auenbruggerplatz 30, 8036 Graz, Austria, Tel. +43 316385 81134; Fax: +43 318385 12678; E-mail: Bernhard. resch@medunigraz.at

Received December 13, 2011; Accepted December 15, 2011; Published December 18, 2011

Citation: Resch B (2012) The Challenge of Early Diagnosis of Bacterial Infection in Neonates. J Neonatal Biol 1:e101. doi:10.4172/2167-0897.1000e101

Copyright: (c) 2012 Resch B. This is an open-access article distributed under the terms of the Creative Commons Attribution License, which permits unrestricted use, distribution, and reproduction in any medium, provided the original author and source are credited. 
the sensitivity and specificity of PCT in the diagnosis of early onset sepsis were reported to be $76 \%$ (range $68-82 \%$ ) and $76 \%(60-87 \%$ ), respectively. As compared to the many other proinflammatory markers there remain still many non-infectious causes of elevated levels, thus, one might argue not to use them to justify continuation of antibiotic therapy in infants without convincing signs of sepsis [10]. Other analyses found a very good accuracy of serum PCT limited by a marked observed statistical heterogeneity [14]. This result again questions the reliability of these tests, and as an individual sepsis marker, none of these mediators reliably enough identifies infected neonates or excludes infection in those at risk [10]. Reasonable, combination tests have been used in studies to increase sensitivity and negative predictive values in order to enhance the likelihood of an abnormal result in the face of uncertainty about the stage of illness at which a neonate is evaluated for suspected infection. But again no panel of sepsis markers prospectively proved to have both excellent sensitivity and good specificity [10].

Up to now every neonatologist is faced with the uncertainty of under- or over- diagnosing bacterial infection, and positive blood cultures are not the "philosopher's stone" per se. As a result many neonates and especially those born preterm receive empiric antibiotic therapy, and the longer they are treated the more resistant pathogens develop in case of unconfirmed infection. If the clinical picture combined with a negative culture result allows justification of the neonate as being not infected antibiotics should be terminated as early as possible. This is especially difficult and on the other hand emergent in preterm infants and especially those with extremely low birth weight who have an increased risk for the development of necrotizing enterocolitis and death in case of prolonged initial empiric antibiotic treatment [15].

What are the goals and challenges for the future? CRP has the advantage of being well characterized in numerous studies and the extensive knowledge on its properties and limitations makes it safer compared to other, newer markers. Still, further research is needed on the topics of the influence of gestational age on kinetics of many of the tested inflammatory markers in infection, non-infectious confounders, and the evaluation of dynamic and gestational age dependent reference values.

\section{References}

1. Stoll BJ, Hansen N (2003) Infections in VLBW infants: studies from the NICHD Neonatal Research Network. Semin Perinatol 27: 293-301.

2. Palazzi DL, Klein JO, Baker CJ (2001) Bacterial sepsis and meningitis. In: Remington JS, Klein JO (eds) Infectious Diseases of the Fetus and Newborn Infant. Philadelphia, PA: WB Saunders.

3. Goldstein B, Giroir B, Randolph A (2005) International pediatric sepsis consensus conference: definitions for sepsis and organ dysfunction in pediatrics. Pediatr Crit Care Med 6: 2-8.

4. American College of Chest Physicians/Society of Critical Care Medicine (1992) Consensus Conference: definitions for sepsis and organ failure and guidelines for the use of innovative therapies in sepsis. Crit Care Med 20: 864-874.

5. Levy MM, Fink MP, Marshall JC, Abraham E, Angus D, et al (2001) SCCM/ ESICM/ACCP/ATS/SIS International Sepsis Definitions Conference. Crit Care Med 31: 1250-1256.

6. Brilli RJ, Goldstein B (2005) Pediatric sepsis definitions: past, present, and future. Pediatr Crit Care Med 6: S6-8

7. Hofer N, Müller W, Resch B (2010) Systemic inflammatory response syndrome (SIRS) definition and correlation with early-onset bacterial infection of the newborn. Arch Dis Child Fetal Neonatal Ed 95: F151.
8. Modi N, Doré CJ, Saraswatula A, Richards M, Bamford KB, et al (2009) A case definition for national and international neonatal bloodstream infection surveillance. Arch Dis Child Fetal Neonatal Ed 94: F8-F12

9. Schelonka RL, Chai MK, Yoder BA, Hensley D, Brockett RM, et al. (1996) Volume of blood required to detect common neonatal pathogens. J Pediatr 129: $275-278$.

10. Benitz WE (2010) Adjunct laboratory tests in the diagnosis of early-onset neonatal sepsis. Clin Perinatol 37: 421-438.

11. Reyna-Figueroa J, Yuri-Toala E, Ortiz-lbarra FJ, Rodríguez-Ramírez E, Limón-Rojas AE (2006) Disparity in the criteria for including patients with neonatal sepsis in scientific medical studies. Are we swimming in a sea without limits?. An Pediatr (Barc) 65: 536-540.

12. Da Silva O, Ohlsson A, Kenyon C (1995) Accuracy of leukocyte indices and C-reactive protein for diagnosis of neonatal sepsis: a critical review. Pediatr Infect Dis J 14: 362-366.

13. Hofer N, Müller W, Resch B (2011) Non-infectious conditions and gestational age influence $\mathrm{C}$-reactive protein values in newborns during the first 3 days of life. Clin Chem Lab Med 49: 297-302.

14. Hofer N, Resch B (2011) CRP in term and preterm newborns. Clin Chim Acta 412: 1888

15. Vouloumanou EK, Plessa E, Karageorgopoulos DE, Mantadakis E, Falagas ME (2011) Serum procalcitonin as a diagnostic marker for neonatal sepsis: a systematic review and meta-analysis. Intensive Care Med 37: 747-762.

16. Cotton CM, Taylor S, Stoll B, Goldberg RN, Hansen NI, et al. and the NICHD Neonatal Research Network (2009) Prolonged duration of initia empiric antibiotic treatment is associated with increased rates of necrotizing enterocolitis and death for extremely low birth weight infants. Pediatrics 123 : 58-66. 